Research Paper

\title{
Neutrophil Suppresses Tumor Cell Proliferation via Fas /Fas Ligand Pathway Mediated Cell Cycle Arrested
}

\author{
Bingwei Sun ${ }^{1}$, Weiting Qin², Mingming Song ${ }^{1}$, Lu Liu ${ }^{3}$, Yao Yu ${ }^{3}$, Xinxin Qi ${ }^{3}$, and Hui Sun ${ }^{3}$ \\ 1. Department of Burns and Plastic Surgery, Suzhou Hospital Affiliated to Nanjing Medical University, Suzhou 215002, Jiangsu Province, China \\ 2. Central Laboratory of Affiliated Hospital, Jiangsu University, Zhenjiang 212001, Jiangsu Province, China \\ 3. Department of Burns and Plastic Surgery, Affiliated Hospital, Jiangsu University, Zhenjiang 212001, Jiangsu Province, China \\ $\triangle$ Corresponding author: Professor Bingwei Sun, MD, PhD, Department of Burns and Plastic Surgery, Suzhou Hospital Affiliated to Nanjing Medical \\ University, Suzhou 215002, Jiangsu Province, China. Telephone: +86 5126236 3018; Fax: +86 5126236 3018; Email: sunbinwe@ujs.edu.cn \\ (c) Ivyspring International Publisher. This is an open access article distributed under the terms of the Creative Commons Attribution (CC BY-NC) license \\ (https://creativecommons.org/licenses/by-nc/4.0/). See http://ivyspring.com/terms for full terms and conditions.
}

Received: 2018.08.16; Accepted: 2018.10.07; Published: 2018.11.28

\begin{abstract}
While neutrophils have dutifully performed their function in injury and infection, the recent works have found that cytotoxicity and/or cytostatic of neutrophils has also been observed in tumor. Till now the molecular players that participate in this neutrophils antitumoral effect remain unclear. In the current study, we find that neutrophils from healthy donors have potent suppression to tumor cell lines by physical contact. Importantly, these suppression activities seem to be cancer cell-specific which is not observed in the normal cells. Further observations show that neutrophils mediated tumor cell lines growth inhibitory effect through early cell cycle arrested. Treatment with an antagonist Fas receptor in A549 cell line or knocking out of the Fas gene in A549 cell line recovers tumor cells cycle and lessen neutrophils anti-tumor effect. The interaction between neutrophils and A549 cell line through Fas ligand /Fas regulates the expression of cell cycle checkpoint proteins, leading to early cell cycle arrest. This phenomenon is also seen in other 3 tumor cell lines. Taken together, our results identified a new role of Fas ligand /Fas interaction in neutrophils antitumoral effect in tumors via arresting cell cycle.
\end{abstract}

Key words: neutrophil, tumor, Fas / Fas ligand, cell proliferation, suppression

\section{Introduction}

Neutrophils are the most abundant leukocytes in the blood and are the first defense against infection and tissue damage [1]. Besides this classical role in antimicrobial function in infection, neutrophils are also found infiltrating in many types of tumors, which may also play a role in tumoral fate. Given that a long progress in tumor growth, early studies suggested that neutrophils would be mere bystanders in tumor since its short longevity [2]. However, more recently it is becoming clear that neutrophils play a critical role in tumor. Although some studies suggest a tumor-promoting role of neutrophils, under certain conditions, they also seem to exert tumor-inhibitory actions $[3,4]$.

The antitumoral activity of neutrophils in in vivo/vitro and clinical trials have been reported and the detail antitumoral action is attributed to direct and indirect function [5]. A study of the cancer-resistant mice SR/CR shows that the antitumoral properties are in fact due to PMN, macrophage and NK cells [6]. In this regard, some more investigations also find that neutrophils from healthy donors have potent cancer-resistant activity [7]. Besides, it is shown that neutrophils could mediate antibody-induced anti-tumor effects in mice with subcutaneous tumors [8]. Furthermore, in 1975, Takasug et.al first showed that peripheral blood PMN possessed direct cytotoxicity against various cultured tumor cell lines and over the years, a large number of reports showed that PMN had cytotoxicity and/or cytostatic effects on tumor in vitro $[7,9,10]$. The published studies above show that neutrophils could be a potential antitumor therapeutic tool.

Tumor suppression resulting from these direct cytotoxic and/or cytostatic effects of neutrophils has been researched and some properties of antitumor 
neutrophils have been described. Superoxide anion or other ROS have been identified as effector molecules in the mechanisms of oxygen dependent antitumoral activity of neoplastic cells by neutrophils [11]. Besides, neutrophils can utilize Fas ligand /Fas to regress tumor cells [12]. And it seems that the antitumoral effect requires physical contact between the immune and malignant cells [7]. However, the mechanisms by which neutrophils exert these effects have not yet been defined.

In this paper, we demonstrate that leukocytes from healthy donors could restrain tumor growth. Additionally, human neutrophils and PBMC cancer resistant activity are investigated and report that the neutrophils are the main character to exert the resistant activity, as other groups have shown, but the mechanism by which neutrophils promote antitumor immunity is poorly understood. And this neutrophils suppression activity is not observed in normal cells which indicate that neutrophils growth inhibitory effect seems to be cancer cell-sepecific. Then we examine the cell cycle and apoptosis of the co-cultured tumor cells and the data show that the role of neutrophils in promoting antitumor immunity is to arrest early tumor cell cycle. In parallel, the indirect contact culture model is applied and the findings suggest that neutrophils antitumoral effect requires direct contact. Based on these findings, we further explore the interaction between the neutrophils and tumor cells and find that the Fas ligand /Fas signal transduction leads to tumor cell cycle arrest with using Fas receptor antagonist and Fas knock out cell lines.

\section{Materials and Methods}

\section{Ethics approval and consent to participate}

This study was approved the Medical Ethical Committee of Jiangsu University. Blood specimens were obtained from the cubital veins of healthy drug-free donors after receiving the written informed consent. Consent for the use of these samples was given by the Medical Ethical Committee of Jiangsu University. All of the experiments were performed in accordance with the approved guidelines.

\section{Materials}

DMEM and Fas receptor antagonists were obtained from thermo scientific (Waltham, MA, USA). Fas receptor agonist was obtained from Biolegend (San Diego, CA, USA). Fetal bovine serum (FBS), protease inhibitor cocktail, Ficoll-Paque, Dextran and RIPA buffer were obtained from Sigma-Aldrich (ST. Louis, MO, USA). Antibodies for western blots were obtained from Cell Signaling Technology (Boston, MA, USA). Antibodies for FCM and apoptosis kits were obtained from BD Bioscience (San Jose, CA, USA). Cell cycle kit was obtained from DAKEWE (Shanghai, China).

\section{Cell culture}

A549 cell line, A431 cell line, Hela cell line and HepG2 cell line were obtained from ATCC and cultured in DMEM with 10\% (vol/vol) FBS. Endothelial cells were isolated as previously described and culture in ECM medium with 10\% (vol/vol) FBS. Cells were maintained in a humidified incubator at $37^{\circ} \mathrm{C}, 5 \% \mathrm{CO}_{2}$.

\section{Isolation of human neutrophils and PBMC}

Human peripheral blood was collected from healthy persons and neutrophils were isolated using Ficoll/ Hypaque centrifugation, as previously described [13]. In brief, blood was mixed with an equal volume of Dextran (3\% in HBSS) and incubated for $30 \mathrm{~min}$ at room temperature. The supernatant was collected and layered on top of Ficoll, following a centrifugation. The neutrophil-containing pellet was resuspended in $3 \mathrm{ml}$ water for $30 \mathrm{~s}$ to facilitate erythrocyte lysis. Isotonicity was restored by the addition of $3 \mathrm{ml} 2 \times$ HBSS. The neutrophil pellet was then washed three times with Hank's balanced salt solution and resuspended in DMEM containing 5\% fetal bovine serum.

For PBMC isolation, whole blood diluted 1:1 with Hank's Balanced Salt Solution was overlaid onto Ficoll separation media and centrifuged at $400 \mathrm{~g}$ for 35 min with no brake. The PBMC at the plasma interface were collected and washed with HBSS twice by centrifugation at $200 \mathrm{~g}$ for $15 \mathrm{~min}$ at room temperature (RT).

\section{Neutrophils migration assay}

Chemotaxis was evaluated according to the previously described[14]. A 24-well microchemotaxis plate was applied, which contained with neutrophil $\left(1 \times 10^{6} / \mathrm{ml}\right)$ in the upper chamber and different chemokines (tumor, supernatant, IL-8) in the lower chamber. Plates were incubated at $37^{\circ} \mathrm{C}$ with $5 \% \mathrm{CO}_{2}$ for $2 \mathrm{~h}$. Then the cells in the lower chamber were collected and the results are presented as the mean number of neutrophils per well. In addition, neutrophils migration also assayed using under agarose neutrophil chemotaxis model [15]. Briefly, agarose solution mixed with medium consisting with $50 \%$ HBSS with $\mathrm{Ca}^{2+}$ and $\mathrm{Mg}^{2+}$ and 50\% RPMI 1640 (20\% heat-inactive FBS). Then pipetted $3 \mathrm{~mL}$ solution into a $35 \mathrm{~mm}$ culture dish and cooled. Till the agarose solution turning solid and cool, three wells were cut into a straight line in the gel. A549 cell were seeded into the middle well and the other two wells were 
seeded with neutrophils. The gel cultured $2 \mathrm{~h}$ at $37^{\circ} \mathrm{C}$ with $5 \% \mathrm{CO}_{2}$. Neutrophils chemotaxisto tumor cells were observed for $50 \mathrm{~min}$ and presented as the distance observing with microscope.

\section{In vitro suppression assay and morphological observation}

Cell proliferation was evaluated by the colorimetric water-soluble tetrazolium salt (CCK8) assay using a cell counting kit- 8 according to the manufacture's instruction. Tumor cells were seeded onto 96-well plate and incubated for $24 \mathrm{~h}$. Then fresh medium containing various concentrations of neutronphils (treated with indicated) were added to the culture plate and co-cultured for another $24 \mathrm{~h}$. The morphological change was observed under an inverted phase-contrast microscope and SEM. Following washing steps to remove the neutrophils and the remaining viable tumor cells number was assessed by measurement of the absorbance at $450 \mathrm{~nm}$ using a microplate reader. Besides, the morphological change was also observed with scanning electron microscope. In brief, the tumor cells were seeded onto a glass slide in the 24-well plate for 24 hours and then treated with neutrophils incubating for another $24 \mathrm{~h}$. Then the cells were fixed with $2.5 \%$ glutaraldehyde in PBS for $15 \mathrm{~min}$. Following washing twice with PBS, the fixed cells were dehydrated with an ascending sequence ethanol. After evaporation of ethanol, the samples were left to dry and then observed under SEM after gold-palladium sputtering.

\section{Cell cycle and apoptosis analysis}

After $24 \mathrm{~h}$ neutrophils co-cultured with A549 (A431, Hela, hepG2) cells in 24-well plate, all cells were collected by trypsinisation and washed with phosphate-buffered saline (PBS). For cell cycle assay, the cells were stained with CD66b, following the cell cycle rapid detection solution was added into the cells. Then the stained cells were analyzed by flow cytometry. Neutrophils were ruled out with CD66b staining and the remaining tumor cells were gated to determine the cell cycle distribution. The cell debris were gated out and the cell populations at G0/G1, S and G2 phases were analysis by using lowjo 7.6.1. For apoptosis assay, an apoptosis detection kit was used according to the manufacture's instruction. In brief, cells were collected and resuspended in $1 \times$ binding buffer at a concentration of $1 \times 10^{6}$ cells $/ \mathrm{ml}$. Then, CD66b, 5ul Annexin-V and 5ul PI were added into the cells suspension and the samples were incubation for $15 \mathrm{~min}$ in the dark. Apoptosis was determined by flow cytometry and Annexin-V positive and PI negative was the cells undergoing apoptosis.

\section{Western blot}

Tumor cells (treated with indicated) were incubated with various concentrations of neutrophils for $24 \mathrm{~h}$ and then the neutrophils were ruled out with CD66b staining and the remaining tumor cells were collected to examine the protein expression. In brief, total cells lysates were obtained and mixed with $3 \times$ SDS buffer, boiled and loaded on 10\% SDS-PAGE gels. Equal amount of protein were separated by SDS-PAGE and transferred to nitrocellulose filters. Non-specific binding was blocked in 3\% BSA in TBS/Triton, followed by incubation with primary antibody $4^{\circ} \mathrm{C}$ overnight. Then the filters were incubated with the appropriate secondary antibody for $1 \mathrm{~h}$ before enhanced chemiluminescence detection. The bands were visualized using ECL reagent.

\section{Fas, Fas ligand expression analysis}

A549 cells were incubated with neutrophils for $24 \mathrm{~h}$ and then all cells were collected. Single cell suspensions were washed twice with ice-cold PBS and resuspended in PBS containing 5\% FBS. Added appropriately conjugated fluorescent (anti-Fas ligand-PE) or purified primary antibody (anti-Fas) and incubated on ice for 30 minutes in the dark. Cells were washed twice with ice-cold PBS following centrifugation at $500 \mathrm{~g}$ for $5 \mathrm{~min}$. In terms of Fas expression, resuspended pellet in PBS with 5\% FBS and added PE goat anti-mouse lgG and incubated on ice in the dark for $30 \mathrm{~min}$ and washed again. Resuspended cell pellet and analyzed with a flow cytometer. In addition, Fas Ligand was also measured using a commercial kit according to the manufacture's recommendations to determine the concentration of Fas Ligand in culture supernatant.

\section{Fas knock out A549, A43 1 cell line}

Fas knock out with lenti-CAS9-sgRNA system was constructed by GeneChem (Shanghai, China). Stably knock out cell lines were generated as previously described [13]. In brief, lentiviral vectors were transfected into tumor cell lines (A549, A431) at indicated MOI $(20,20)$ in the presence of $5 \mu \mathrm{g} / \mathrm{ml}$ polybrene. Replaced fresh medium after 12 hours culture. The relevant empty lentivectors were used as negative control. Western blot was used to determine the expression of Fas.

\section{Human XL cytokine proteome array}

The human XL cytokine proteome array was performed according to manufacturer's instructions. Neutrophil co-cultured (direct or indirect contact) with A549 for $24 \mathrm{~h}$, then collected the culture supernates for the human XL cytokine array. ImageJ 
software was used to determine the pixel density in each spot of the array.

\section{Statistical analysis}

All statistical analyses were performed with the GraphPad Prism Software (version 4). Results were expressed as the mean \pm SD. One-way ANOVA for multiple groups and Student's $t$ test for 2 groups were applied for statistical analysis. Statistical significance was set at $p \leq 0.05$.

\section{Results}

\section{Neutrophils migrate to tumor cells}

Lots of studies have been described that cancer cells could produce neutrophil chemoattractants [16, 17]. When co-cultured neutrophils with tumor cells, neutrophils gradually migrated to tumor cells and after $24 \mathrm{~h}$ incubation, neutrophils surrounded the tumor cells was observed (Fig. 1a, b). To test the chemotactic function of tumor cells, we utilized transwell assay and under agarose neutrophil chemotaxis model in A549 cells. After 2 h incubation, in transwell assay neutrophils were collected from the bottom and quantified. As seen in fig 1e, compared with the control group, A549 cell and its supernatant could mount more neutrophils migration. The similar results were also observed in the under agarose neutrophil chemotaxis model (Fig. 1c, d). Besides, we detected the A549 cell supernatant for the production of neutrophil chemoattractants using protein chip. As shown in fig. 1f, g several relevant cytokines were detected and the level of each cytokine was higher than the control group. These data above indicated that chemokines expressed by A549 cells could induce neutrophil migration toward the tumor situ.

\section{Neutrophils induce an antitumoral effect on tumor cells}

We first performed CCK8 assay to determine the effect of leukocytes on A549 cells growth in vitro. Leukocytes from healthy donors were co-cultured with A549 cells at different effector to target (E: T) cell ratios. As seen in fig 2a, leukocytes detectably contributed the anti-tumor function. To further examine which cell contributed the result, a role of neutrophils and PBMC were detected. As shown in Figure $2 \mathrm{a}, \mathrm{b}$, a role of PBMC was ruled out, whereas neutrophils did play a role in restraining tumor growth which was in a dose and time dependent way. Strikingly, neutrophils also exhibited a broad antitumoral effect to other three different tumor cells (Fig. 2c). And this phenomena was not observed in normal cells which indicated neutrophil cytotoxicity or (and) cytostatic effects might be cancer cell specific
(Fig.2h). Further, to research whether the growth inhibitory effect of neutrophils was mediated by induction of an apoptotic cell death or cell cycle arrest, the apoptosis and cell cycle assay were applied. A549 cells were treated with neutrophils for $24 \mathrm{~h}$, the cell apoptosis and the distribution of cells in each phase of the cell cycle were analyzed by flow cytometry (Fig. $2 \mathrm{~d}, \mathrm{e}, \mathrm{f}, \mathrm{g}$ ). Treated with neutrophils, there were no significant changes in the apoptosis of tumor cells in $24 \mathrm{~h}$. However, treatment with neutrophils, tumor cells were accumulated in G0/G1 phase after $24 \mathrm{~h}$ incubation. The phenomena were also observed in other three tumor cell lines (Fig. S4). These results suggested that neutrophils suppressed cell growth through arrest of early cell cycle progression in G0/G1 phase.

\section{Direct contact is required for the growth inhibitory effect of neutrophils}

The morphological changes by neutrophilstreated A549 cells were observed under SEM as illustrated in Figure $3 \mathrm{~h}$ that the neutrophils surrounded along the tumor cells. In order to survey whether the direct contact was required for neutrophils anti-tumor activity, separating A549 cells from neutrophils with transwell. The neutrophils antitumoral effect was largely abrogated as shown in Figure 3a. In parallel, the apoptosis and cell cycle distribution were also examined using a transwell model (Fig. 3d, e, f and g). Similarly, there was little change of apoptosis and the cell cycle distribution in transwell group when compared with control group. In addition, we further set out to determine tumor cells growth when wiping off the co-cultured neutrophils. Tumor cells growth were recovered after $24 \mathrm{~h}$ following culture (Fig. 3b). Besides, treated with neutrophils lysate, there was a consistence result with above that the supernatant of lysate had little effect on tumor cells, whereas the sediment of lysate did have potent effect on tumor cells (Fig. 3c), which indirectly indicated neutrophils suppression of tumor cells required direct contact. In light of our observation that neutrophils could restrain the tumor cells growth in direct contact, we reasoned that the interaction between A549 and neutrophils is the key point.

\section{Neutrophils arrest cell cycle of tumor cells}

The effect of neutrophils on cell cycle distribution of A549 cells was examined in vitro. A549 cells were treated with various concentrations of neutrophils for $24 \mathrm{~h}$ and results were analyzed by FACS. Consistent with the above, neutrophils restrained the progression of A549 cells and the effect was in a concentration dependent way (Fig. 4a, b). 


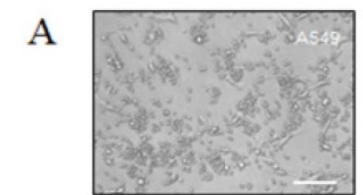

B

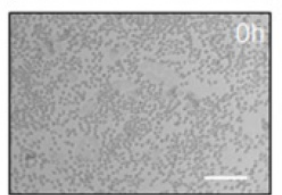

C

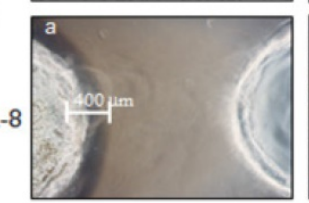

D

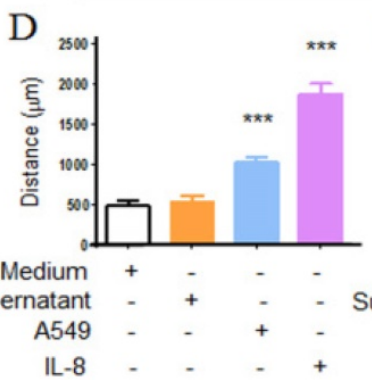

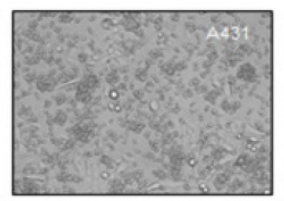
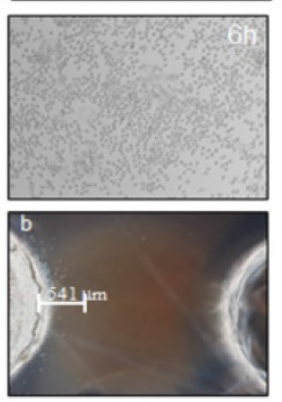

$\mathrm{E}$

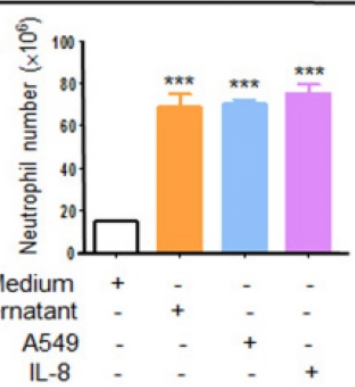

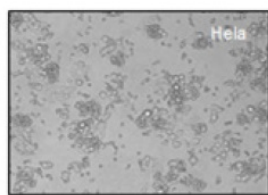
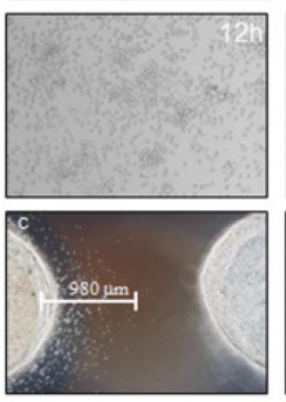

F 40000

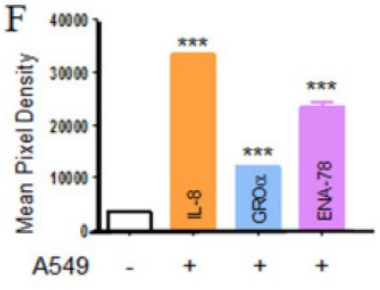

G

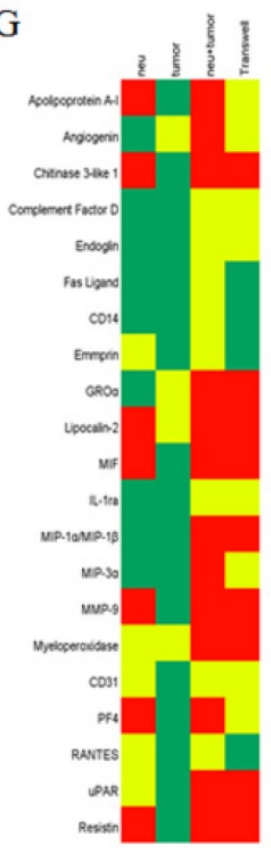

Figure 1. Neutrophils migrates to tumor cells. Purified neutrophils from healthy adults migrating to tumor cells were observed under inverted phase-contrast microscope (a) and the progress was monitored by using living cell station (b), scar bar, $200 \mu$ m. Neutrophils chemotaxis toward A549 cells was assayed by using under agarosechemotaxis model or transwell model. In under agarosechemotaxis model, the results were presented by the migration distance after 2 hours (c, $d$ ). In transwell model, cells were counted from the bottom of transwell plate after 2 hours (e). The production of IL-8, GRO $\alpha$, ENA-78 by A549 cell lines was measured by proteome array $(f, g)$. $a=$ medium; $b=$ tumor supernatant; $c=$ tumor; $d=I L-8$. Data are representative of four $(a, d, e)$ or three $(b, f, g)$ independent experiments. Mean and SD are presented. $* * * P<0.001$, compared with control group (ANOVA with Tukey's test).

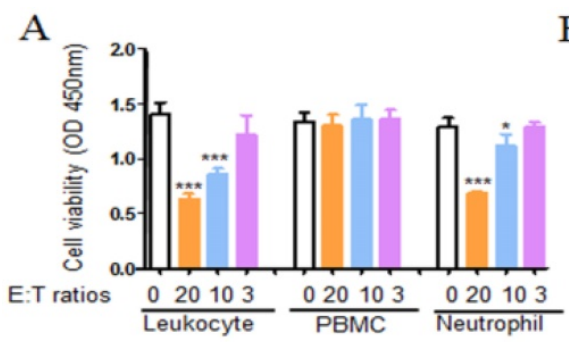

D

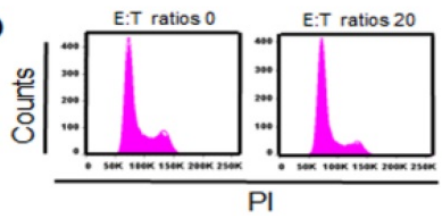

F

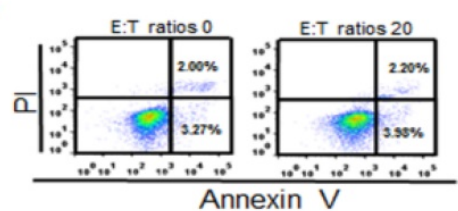

B

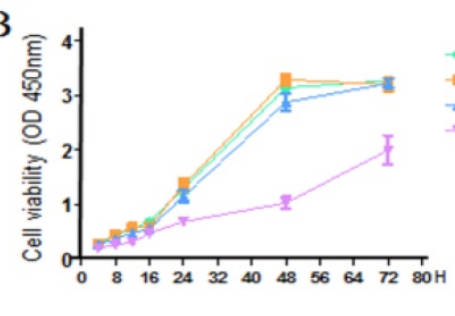

E

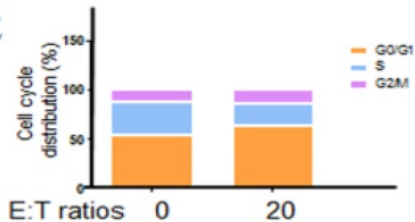

G

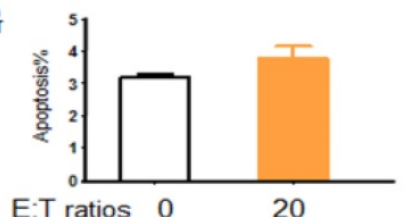

$\mathrm{C}$

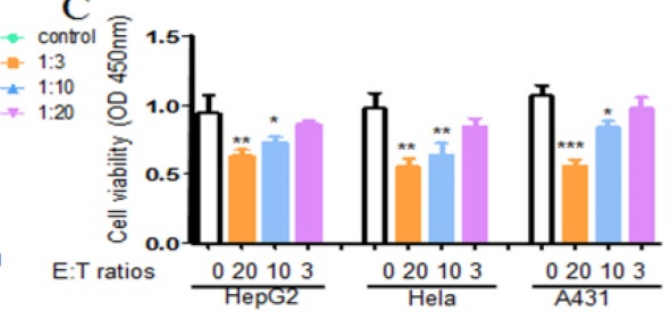

$\mathrm{H}$

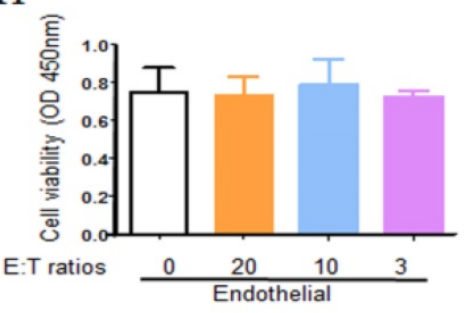

Figure 2. Neutrophils induce an antitumoral effect on tumor cells. A549 (Hela, HepG2, A431, endothelial) cells were seeded onto 96 -well or 24-well plate and incubated for $24 \mathrm{~h}$. Leukocyte, PBMC and neutrophils (treated as indicated) isolated from healthy adults were cocultured with A549 cells at 20:1, 10:1 and 3:1 neutrophil to tumor cell ratio for different time. Following overnight incubation, the antitumoral effects of leukocytes, PBMC and neutrophils were measured using cck8 assay kit (a). Neutrophils co-culture with A549 for 24, 48, 72 hours and the cytotoxicity or (and) cytostatic effects was assessed by cck8 assay kit (b). The antitumoral effect of neutrophils to other tumor cells (Hela, HepG2, and A431) and endothelial cells were also detected using CCK8 assay (c, h). Cell cycle and apoptosis of A549 were assayed using flow cytometry ( $d$, e, f, and g). Data are representative of four independent experiments. Mean and SD are presented. Statistical analysis was performed by one way ANOVA; $* * * p<0.001$, $* * p<0.01, * p<0.05$, compared with control group. 

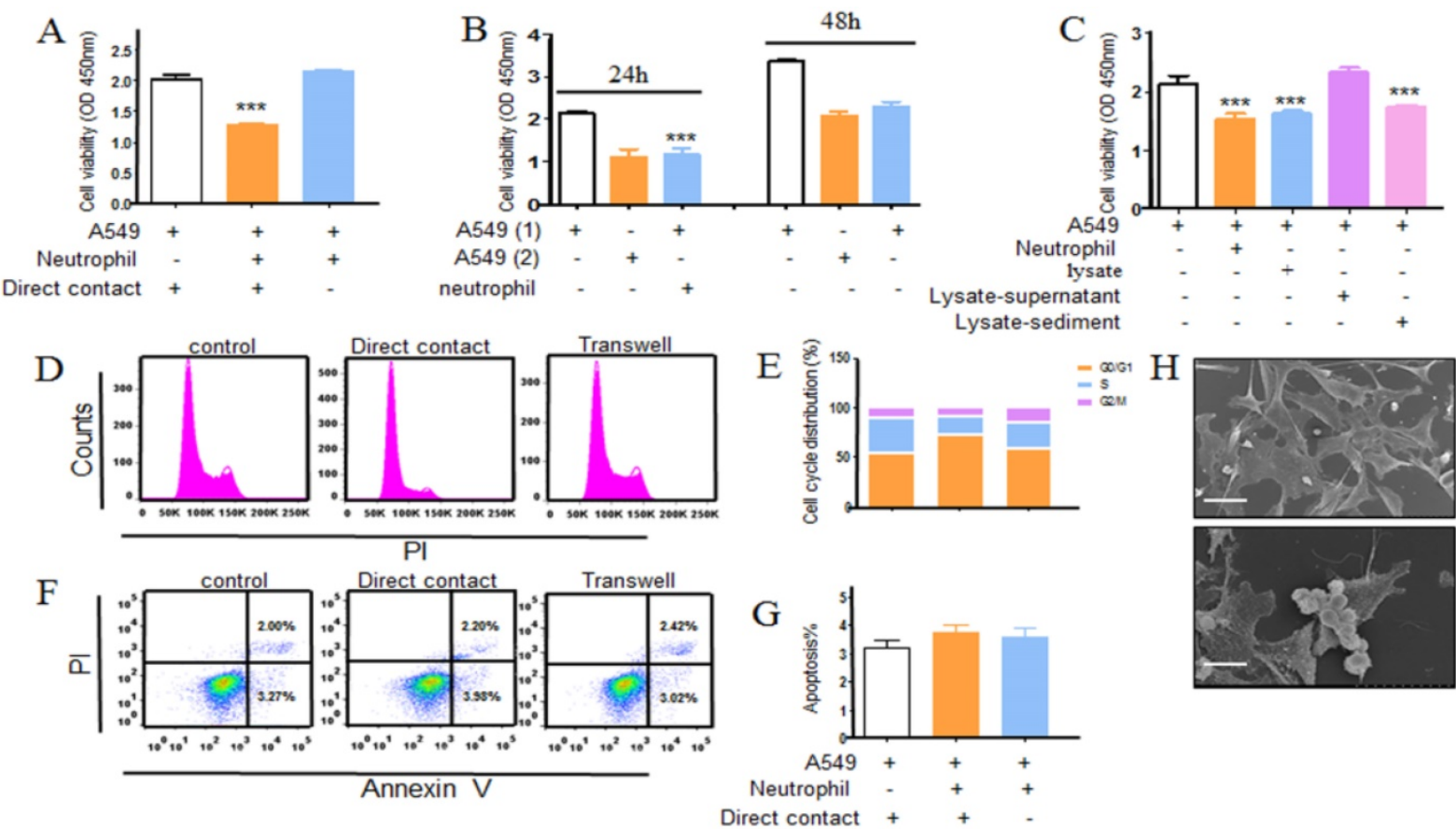

Figure 3. Direct contact is required for the growth inhibitory effect of neutrophils. Neutrophil anticancer effect was assayed by indirectly co-incubating A549 cancer cells with human neutrophils from healthy donors at effector to target (E:T) cell ratio of $20: 1$ using transwell model. Cells were incubated at $37^{\circ} \mathrm{C}$ in a humidified $5 \% \mathrm{CO}_{2}$ incubator for $24 \mathrm{~h}$. Following washing steps to remove the neutrophils and the remaining viable tumor cells number was assessed by using CCK8 kit (a). Cell cycle and apoptosis of A549 were assayed using flow cytometry (d, e, f, g). In addition, we further seted out to determine tumor cells growth when wiping off the co-cultured neutrophils. After 24 hours further culturing, tumor cells growth was examined using CCK8 kit (b). In parallel, neutrophil lysate was also used in the antitumor assay and the result was measured using CCK8 assay (c). Neutrophils surrounded along tumor cells was observed under SEM (h), scale bar, 20 um. Data are representative of four $(\mathrm{a}-\mathrm{g})$ or three $(\mathrm{h})$ independent experiments. Mean and SD are presented. Statistical analysis was performed by one way ANOVA; $* * * P<0.001$, compared with control group.

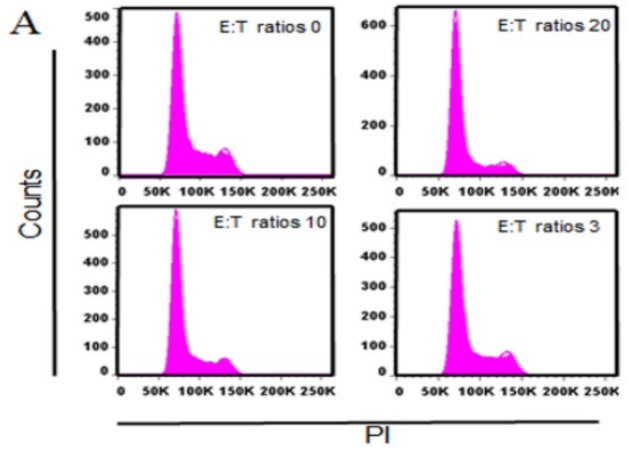

B

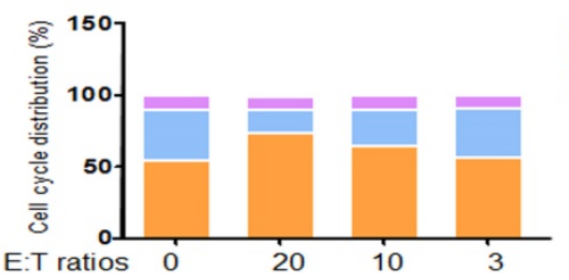

C

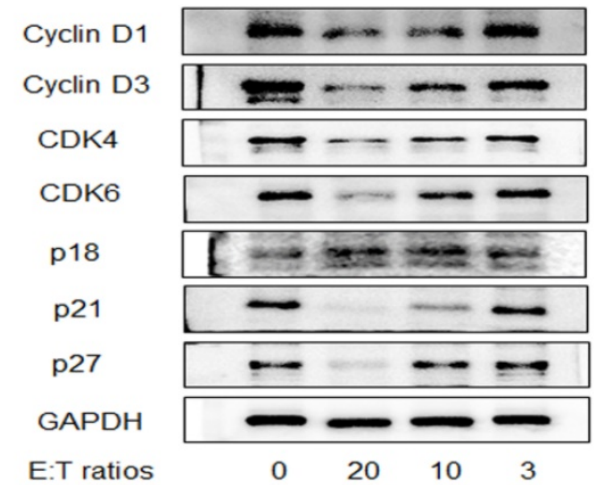

$\mathrm{D}$

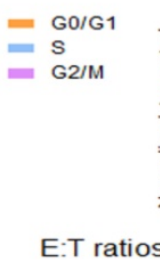

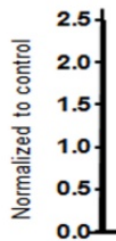

2.5
2.0
1.5
1.0
0.5
0.0

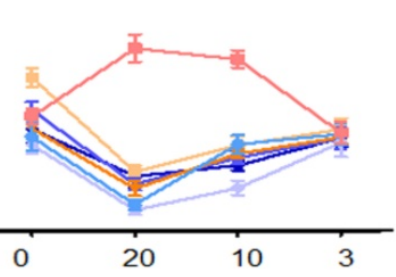

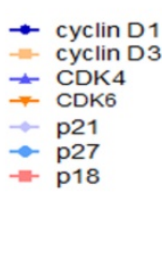

Figure 4.Neutrophils arrest cell cycle of tumor cells. Neutrophils co-incubated with A549 cells at E:T ratios of 20:1, 10:1 and 3:1. Using cell cycle rapid solution to determine the cell cycle distribution, which was analyzed by flow cytometry. The representative images and detail data were shown in a, b. The related cell cycle regulation proteins were evaluated by western blot $(c, d)$. Data are representative of four independent experiments. Mean and SD are presented. Statistical analysis was performed by one way ANOVA; $* * *<<0.001$, compared with control group.

Cell cycle progression was regulated by the level of various cyclins, the activity of cyclin-dependent kinases and the CDK inhibitors [18]. A549 cells were challenged with various concentrations of neutrophils for $24 \mathrm{~h}$ and the expressions of relevant proteins were examined by western blot. Cyclin D1, D3 protein 
levels were diminished after neutrophils treatment (Fig. 4c, d). Neutrophils also resulted in a reduction in CDK-4 and CDK-6 levels (Fig. 4c, d). These findings were consistent with the reduction of $S$ populations from the FASC analysis. In addition the CDK inhibitors were also surveyed. p18, a CDK inhibitor of the p16 znk4 family, forms a stable binary complex with CDK6 and binds CDK4 weakly. p18 has also been shown to inhibit the expression of CDK6 in vitro. Here we found that A549 cells p18 level rose significantly by $24 \mathrm{~h}$ neutrophils treatment. Unexpectedly, neutrophils contributed a strong reduction in the other CDK inhibitors p21 and p27 (Fig. 4c, d). p21 and p27 have a dual function in the cell cycle: inhibition of CDK-cyclin formation, particularly cyclin E-cdk2, as well as facilitating the assembly of cyclinD-cdk $4 / 6$ complexes [19].

\section{Neutrophils arrest cell cycle of tumor cells via Fas Ligand/ Fas pathway}

Neutrophil, as the first line of host defense, could wipe out pathogens through phagocytosis, extracellular degranulation, respiratory burst or spreading of extracellular traps [20]. To prospectively determine whether degranulation, respiratory burst or NETs executed neutrophil anti-tumor activity, NADPH oxidase inhibitor diphenyleneiodonium (DPI), neutrophil elastase inhibitor alvelestat and supernatant from FMLP stimulated neutrophils were applied in the antitumoral assay. Data (Fig. S1A and B) showed that treatment with stimulated neutrophils supernatant containing lots of granules, there was no significant changes in tumor cells cell cycle distribution. In parallel, as shown in Fig. S1C, neither DPI nor alvelestat did significantly alter neutrophils mediated A549 suppression effect, which implied that neutronphils respiratory burst or super oxide production or NETs were not participated in this anti-tumor effect observed.

As shown in proteome array, after co-culture some supernatant cytokines changed a lot. In it, some molecules (such as IL-8, Fas ligand et.al) attracted our attention, which were highly related with cell proliferation. Then the corresponded antagonist was used to determine whether these molecules displayed a role in neutrophil anti-tumor activity. We observed that IL-8 receptor inhibitor did not significantly alter neutrophil mediated A549 cells early cell cycle arrest, whereas engaging Fas antagonist on neutrophils and A549 co-culture resulted in partially growth recovered, indicating the Fas signaling pathway might play a critical role in this neutrophils early cell cycle arrest process (Fig. 5d, Fig. S2D). And this result was also observed in other 3 tumor cells (Fig. S4). In addition, treated with Fas receptor agonist could also lead to growth arrest (Fig. 5d, Fig. S2D). Further, the apoptosis was measured and as shown in Figure 5e, Additional file 2: Figure S2E, there was no significant change when treated with Fas antagonist and a slight increase was observed in Fas agonist treated tumor cells. Here we demonstrated that the proliferation of Fas-knock out A549 cells was partially restored when co-culture with neutrophils (Fig. 5d, Fig.S2A). And this result was also observed in A431 cell lines (Fig. $5 d$, Fig. S2B). Consistent with above results, there was also no significant changes in neutrophils stimulated Fas-knock out tumor cells apoptosis (Fig.5e, Fig. S2C). Besides, the levels of Fas and Fas ligand were detected using flow cytometry and ELASA (Fig. 5c, Fig. S3), which was in accordance with above results that a higher level of Fas ligand of neutrophils was seen in co-culture group and no significant change of Fas of tumor cells was occurred in each group. Following the main cell cycle regulation proteins were examined (Fig. 5f, g) and found that the level of cyclins (cyclin D1, D3) and CDK (CDK4/6) in tumor cells of Fas-agonist group were significantly lower than that of A549 cells from control group and vice versa.

\section{Discussion}

The significant improvement in cancer therapy achieved over recent decades has gradually come about through immunotherapy, which is based mostly on the properties of the adaptive immune system (i.e. B and T lymphocytes) and some components of the innate systems (like NK cells, macrophages or complement proteins)[21, 22]. Despite the majority of immunotherapy trails performed to date in cancer patients having involved $\mathrm{T}$ cells, it is clear from a number of recent studies that other innate immune cell types can induce effective anti-tumor response $[23,24]$. Intriguing, neutrophils, as the main character of the innate immune system, have rarely been considered as a sound tool against tumor and the role as well as the concrete mechanism of neutrophils in tumor suppression has not yet been well defined[24, 25].

Till now, the research thus far on the role of neutrophils in cancer has focused largely on these tumor associated neutrophils, which vary depending on the tumor context [26, 27]. The infiltration and effects of neutrophils within a tumor are due to the intrinsic properties of both tumor and host [26, 28, 29]. Thus, neutrophil may be a double-edged sword that can display both tumor promoting and inhibitory effects. Recent research have demonstrated that using neutrophils from a strain of naturally cancer-restrain mice could regress the tumor growth[6, 30, 31]. In addition, neutrophils from healthy human donors also show an excellent cytotoxicity to the tumors in 
vitro[7]. We speculated that under certain circumstances neutrophils exhibit a powerful antitumor effect.

The results from the present study showed that neutrophils suppressed the growth of tumor, while this was not the first report to show that. Data from this study extended the previous results that neutrophils mediated tumor suppression via Fas/Fas ligand pathway. In addition, we have shown that the suppression was to arrest the tumor cells cell cycle and the interaction between Fas $\mathrm{L}$ and Fas was involved in this process.

In the current study, we demonstrated that neutrophils from some healthy donors exert highly suppression effect to tumor cells, exhibiting $70 \%$ growth inhibitory effect at 20:1 effector-to-target cell ratio after $24 \mathrm{~h}$ co-culture. Four human cancer cell lines including cells from A549, A431, Hela and HepG2 were used as the target cells and above neutrophils antitumor activity could be seen in each of them. Importantly, these neutrophils mediated antitumor activity is specific to tumor cells since the neutrophils displayed little effect against normal cells. This observation suggests that neutrophils may serve as a safeguard protecting host from cancer. Within kinds of human and murine tumor, neutrophils are found to infiltration in it $[2,32]$. Consistent with this, we observed that neutrophils migrated to the tumor site and accumulated around the tumor cells in the co-culture dynamic video and this phenomenon was also seen with the invert-phase microscope and SEM. It has been described a number of lung cancer could produce neutrophil chemokines, such as IL-8, GRO $\alpha$ et.al. In terms of A549 cell line, the chemokines were determined with protein chip and results were shown in Figure $1 \mathrm{~F}, \mathrm{G}$. With these chemoattractants, neutrophils could migrate to the tumor site. As seen in Figure $1 \mathrm{E}$, neutrophils trans-migrated the transwell membrane to the lower chamber, where there were various chemokines. In parallel, using under agarose neutrophils chemotaxis model, the similar results were also observed (Figure $1 \mathrm{C}, \mathrm{D}$ ). These results suggest that neutrophils can detect the presence of tumor and migrate to the tumor site, leading a suppression of the tumor cells.
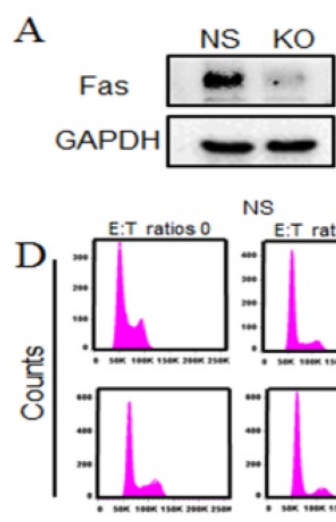

NS

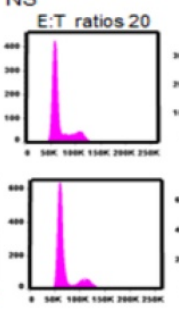

A549+Neu
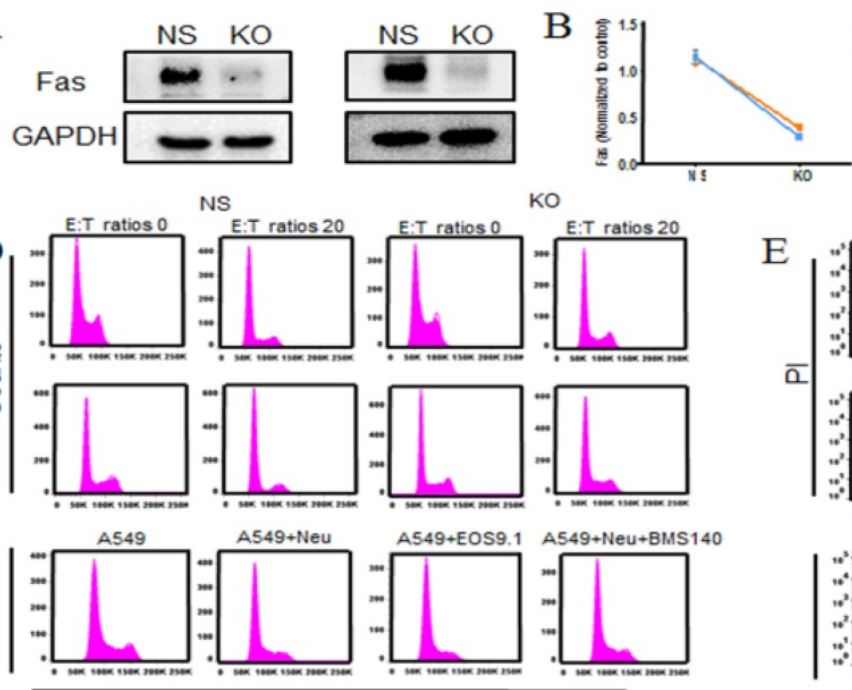

$\mathrm{KO}$
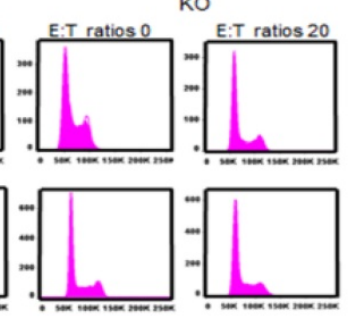

A549+EOS9.1 A549+Neu+BMS140
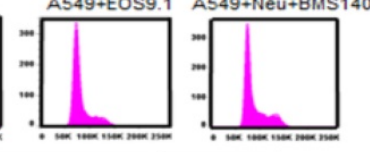

$\mathrm{PI}$

F

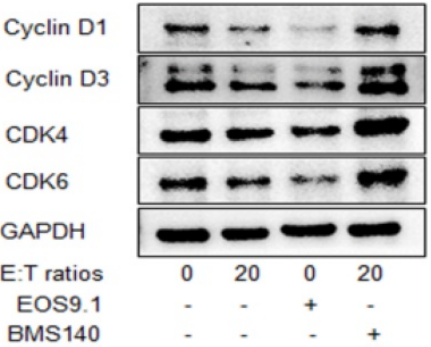

G
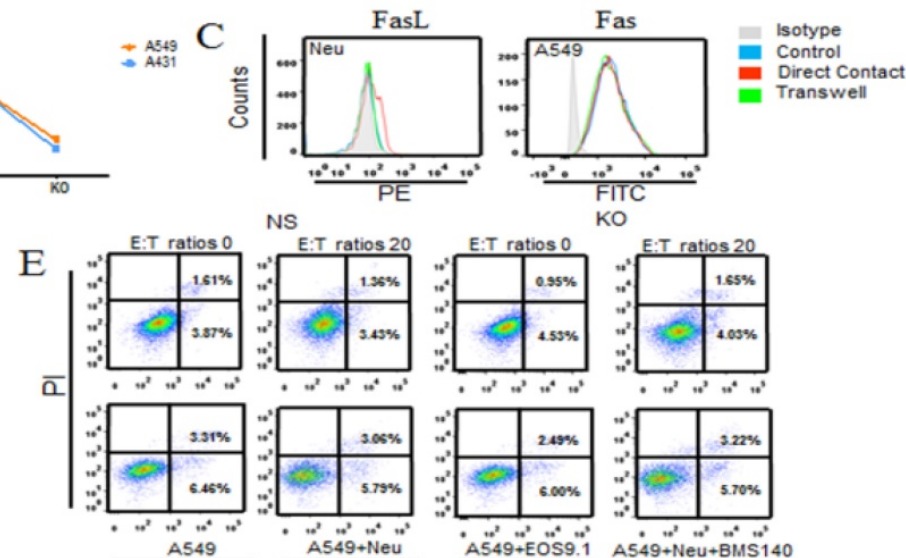

$\mathrm{KO}$
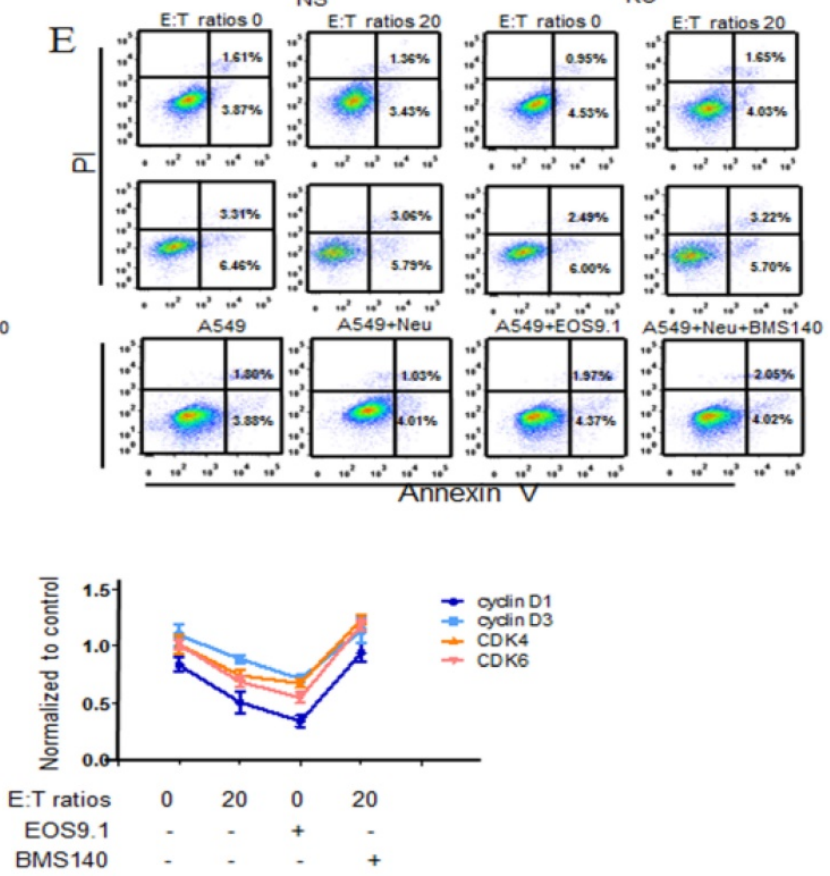

Figure 5. Neutrophils arrest cell cycle of tumor cells via Fas/ Fas ligand pathway. A549 cancer cells co-incubated with human neutrophils from healthy donors in the presence or absence of the Fas antagonist (BMS140) and agonist (EOS9.1) at effector to target (E:T) cell ratio of 20:1. Cell cycle and apoptosis of A549 cells were detected by flow cytometry $(\mathrm{d}, \mathrm{e})$. Fas $\mathrm{L}$ and Fas expression level were further examined by using flow cytometry (c). The relevant cell cycle regulation proteins were evaluated by western blot (f, $g$ ). Fas was knock out in A549 cells. The relevant empty lentivectors were used to induce control nonspecific (NS) expressing cells. Expression of Fas in knock out A549 cells was detected by western blot (a, b). Knock out tumor cells (A549, A431) co-incubated with neutrophils for 24 hours at E:T ratio of 20:1, cell cycle and apoptosis of tumor cells were examined (d, e). Data are representative of four independent experiments. Mean and SD are presented. Statistical analysis was performed by one way ANOVA; *** $<<0.001$, compared with control group. 
In general, the ability of neutrophils to suppress tumor cells could be attributed to the inhibition of proliferation, promoting apoptosis or both. Various in vitro studies have observed this suppression effect, while there is little research coming into the cell proliferation or apoptosis. Our further study indicated that neutrophils suppressing tumor in vitro was mainly due to early cell cycle arrest. Cell cycle, the process by which cells progress and divide, lies at the heart of cancer. Many sources have linked irregularities in the G1 phase or the G1/S checkpoint to uncontrolled growth of tumor and many cancers including breast and skin cancers have been prevented from proliferating by causing the tumor cells to enter G1 cell cycle arrest [33, 34]. Neutrophils exerted their growth arrest on treated tumor cells by accumulating cells at G0/G1 phase with increased p18 level and decreased cyclin D1, D3, CDK4/6 expression, implying that neutrophils may interfere with protein synthesis of tumor cells that halting their progression from G1 to S phase during their cell cycle and preventing the cells from dividing and spreading. Besides, one of the most striking phenomenon about the anti-tumor cytotoxic effect of PMN, according to the collected literature, is the need for an intimate, physical contact between the PMN and the target tumor cell. In vivo experiments, to elicit an antitumor immune response, tumor cell lines undertaken genetic modification to produce cytokines or chemokines demonstrated close contact between PMNs. This phenomenon were observed when transferring G-CSF [35] and IL-2 [36] gene in murine models of adenocarcinoma. In vitro observations, some already ancient $[11,37]$ and from other experimental models in which it is demonstrated that a direct contact is required as separating the tumor cells from the PMN with transwells largely abrogates the killing effect [7, 38, 39]. The same characteristic has also been described in the mechanisms of cellular antibody dependent cytotoxicity (ADCC), produced by PMNs, as demonstrated by multiple publications [40-46]. Consistence with previous results we also found that separate tumor cells from neutrophils with transwell largely abrogated the restrain effect, which implied that the antitumoral effect of neutrophils requires physical contact between the immune cells and malignant cells.

Previous reports have shown that $\mathrm{T}$ cell respond to tumor specific antigen and mediate antitumor responses via expression of IFN- $\gamma$ and granzymes[47] and the involvement mechanisms that neutrophils against bacteria are phagocytosis, release of antimicrobial substances and the formation of neutrophil extracellular traps [20, 48, 49]. Further, research on these released mediates functions in neutrophils antitumoral effects were undertaken. And our findings suggested that neutrophils degranulation, respiratory burst or NETs may be not required for the anticancer effect observed. Then the molecular mechanism by which neutrophils executed these cytostatic effects was studied and here our findings suggested that neutrophils Fas ligand expression interacting with tumor cells Fas resulted in the neutrophils mediated antitumoral activity. Studies in the decades have demonstrated that PMN is related to the rejection of cancer cells expression FasL (CD95L). Cancer cell lines transfected with CD95L gene have shown a suppression of tumor cells with a massive infiltration of PMN and monocytes [50-57]. Depletion of PMN in vivo, the effect of rejection of CD95L+ tumor has reduced which suggests a role of PMN in tumor cell suppression [51]. Further, the cytotoxicity of PMN against colon carcinoma cells transfected with CD95L gene was observed both in vivo and in vitro [52]. The local anti-tumoral effect, mediated mainly by PMN, is also achieved when the cells transfected with CD95L gene are non-malignant fibroblasts mixed with untransfected cancer cells [58]. In addition the simultaneous CD95L and GM-CSF gene transfection into Lewis lung carcinoma cells also suppressed their growth in vivo via massive PMN infiltration [59]. The antitumoral effect achieved by p53 gene transfer, due to its upregulation of CD95L in human cancer and its chemotactic properties toward neutrophils, indirectly indicate the effect of CD95L [60]. Otherwise, CD95L expression on tumor cells is also implicated in tumor evasion of immune surveillance. The high expression of FasL on melanoma cells leads to neutrophil inactivation and assists tumor development and local transfer of PMA-treated neutrophils delayed tumor formation by melanoma cells [61]. In this work, neutrophils function different depend on the expression level of tumor CD95L and the activity of neutrophils in the tumor-bearing mice. Above works addressed the effect of CD95L on tumor [61]. Besides, published reports have suggested that neutrophils via Fas ligand induce tumor cells apoptosis [62]. Intriguing, our research suggested that Fas L signals can partially regulate cell cycle proteins and prevent tumor cells expansion in vitro. When a Fas receptor engages with its ligand, a caspase cascade is initiated, which in most cases leads to apoptosis of $\mathrm{Fas}^{+}$cells [63]. Moreover, accumulating data indicate that Fas signal may modulate other cell process in addition to its well-known death-triggering capability. Engaging FasL on T cells in culture results in growth arrest [64]. Dendritic cells (DC) maturation to release IL-1 $\beta$ and interferon- $\gamma$ during DC-T cell cognate interactions is induced by Fas engagement[65]. In terms of this study, considering the neutrophils seeding time and 
concentration may explain this result. The published report seeded neutrophils when tumor cells attachment and formation of a confluent monolayer which was in the later stage of tumor logarithm stage and the concentration of seeded neutrophils was twice as many as our present study used [62]. We have shown here that Fas agonist can restrain A549 cells proliferation. Furthermore, neutrophils arresting A549 cells cell cycle was partially restored by Fas antagonist engagement. In parallel, our data on Fas-knock out tumor cells indicates that FasL engagement may tonically promote neutrophilsmediated tumor cells suppression.

In conclusion, this work overall characterizes the mechanism by which neutrophils suppress tumor cells via arresting cell cycle in vitro. Our data in this report show that neutrophils gradually accumulate around tumor cells after co-incubation. Such interaction may result in more contact with tumor cells and reach the anti-tumor activity level in early time. Neutrophils are versatile, plastic cells that response to kinds of tumors influences with the expression of distinct transcriptional programs and functions. The available information suggests that neutrophils infiltrate in tumors acquire varieties properties to affect tumor progression. The identification of mechanism and molecular associated with neutrophils mediated antitumoral effect provides a basis for tumor therapeutic strategies.

\section{Supplementary Material}

Supplementary figures and tables.

http://www.ijbs.com/v14p2103s1.pdf

\section{Acknowledgements}

This study was supported by the National Natural Science Foundation of China, No. 81071546, No. 81272148 and No. 81471903 ; by the Jiangsu Natural Science Foundation, No. BE2017695.

\section{Competing Interests}

The authors have declared that no competing interest exists.

\section{References}

1. Borregaard N. Neutrophils, from marrow to microbes. Immunity. 2010;33(5):657-670.

2. Uribe-Querol E, Rosales C. Neutrophils in Cancer: Two Sides of the Same Coin. J Immunol Res. 2015;2015:983698

3. Mantovani A, Allavena P, Sica A, et al. Cancer-related inflammation. Nature. 2008;454(7203):436-444.

4. Clark RA, Szot S. The myeloperoxidase-hydrogen peroxide-halide system as effector of neutrophil-mediated tumor cell cytotoxicity. J Immunol. 1981;126(4):1295-1301.

5. Souto JC, Vila L, Bru A. Polymorphonuclear neutrophils and cancer: intense and sustained neutrophilia as a treatment against solid tumors. Med Res Rev. 2011;31(3):311-363.

6. Cui Z, Willingham MC, Hicks AM, et al. Spontaneous regression of advanced cancer: identification of a unique genetically determined, age-dependent trait in mice. Proc Natl Acad Sci U S A. 2003;100(11):6682-6687.
7. Yan J, Kloecker G, Fleming C, et al. Human polymorphonuclear neutrophils specifically recognize and kill cancerous cells. Oncoimmunology. 2014;3(7):e950163.

8. Albanesi M, Mancardi DA, Jonsson F, et al. Neutrophils mediate antibody-induced antitumor effects in mice. Blood. 2013;122(18):3160-3164.

9. Takasugi M, Akira D, Kinoshita K. Granulocytes as effectors in cell-mediated cytotoxicity of adherent target cells. Cancer Res. 1975;35(8):2169-2176.

10. Shau H. Characteristics and mechanism of neutrophil-mediated cytostasis induced by tumor necrosis factor. J Immunol. 1988;141(1):234-240.

11. Dallegri F, Ottonello L, Ballestrero A, et al. Tumor cell lysis by activated human neutrophils: analysis of neutrophil-delivered oxidative attack and role of leukocyte function-associated antigen 1 . Inflammation. 1991;15(1):15-30.

12. Springer ML, Kraft PE, Blau HM. Inhibition of solid tumor growth by Fas ligand-expressing myoblasts. Somat Cell Mol Genet. 1998;24(5):281-289.

13. Liu X, Ma B, Malik AB, et al. Bidirectional regulation of neutrophil migration by mitogen-activated protein kinases. Nat Immunol. 2012;13(5):457-464.

14. Fan J, Malik AB. Toll-like receptor-4 (TLR4) signaling augments chemokine-induced neutrophil migration by modulating cell surface expression of chemokine receptors. Nat Med. 2003;9(3):315-321.

15. Wang X, Qin W, Xu X, et al. Endotoxin-induced autocrine ATP signaling inhibits neutrophil chemotaxis through enhancing myosin light chain phosphorylation. Proc Natl Acad Sci U S A. 2017;114(17):4483-4488.

16. Viola A, Sarukhan A, Bronte V, et al. The pros and cons of chemokines in tumor immunology. Trends Immunol. 2012;33(10):496-504.

17. Dubinett SM, Lee JM, Sharma S, et al. Chemokines: can effector cells be redirected to the site of the tumor? Cancer J. 2010;16(4):325-335.

18. Malumbres $M$, Barbacid M. Cell cycle, CDKs and cancer: a changing paradigm. Nat Rev Cancer. 2009;9(3):153-166.

19. Sherr CJ, Roberts JM. CDK inhibitors: positive and negative regulators of G1-phase progression. Genes Dev. 1999;13(12):1501-1512.

20. Kolaczkowska E, Kubes P. Neutrophil recruitment and function in health and inflammation. Nat Rev Immunol. 2013;13(3):159-175.

21. Bhardwaj N. Harnessing the immune system to treat cancer. J Clin Invest. 2007;117(5):1130-1136.

22. Finn OJ. Cancer immunology. N Engl J Med. 2008;358(25):2704-2715.

23. Yonezawa A, Chester C, Rajasekaran N, et al. Harnessing the innate immune system to treat cancer: enhancement of antibody-dependent cellular cytotoxicity with anti-CD137 Ab. Chin Clin Oncol. 2016;5(1):5.

24. Di Carlo E, Forni G, Lollini $P$, et al. The intriguing role of polymorphonuclear neutrophils in antitumor reactions. Blood. 2001;97(2):339-345.

25. Sionov RV, Fridlender ZG, Granot Z. The Multifaceted Roles Neutrophils Play in the Tumor Microenvironment. Cancer Microenviron. 2015;8(3):125-158

26. Fridlender ZG, Albelda SM. Tumor-associated neutrophils: friend or foe? Carcinogenesis. 2012;33(5):949-955.

27. Fridlender ZG, Sun J, Kim S, et al. Polarization of tumor-associated neutrophil phenotype by TGF-beta: "N1" versus "N2" TAN. Cancer Cell. 2009;16(3):183-194.

28. Kruger P, Saffarzadeh M, Weber AN, et al. Neutrophils: Between host defence, immune modulation, and tissue injury. PLoS Pathog. 2015;11(3):e1004651.

29. Swierczak A, Mouchemore KA, Hamilton JA, et al. Neutrophils: important contributors to tumor progression and metastasis. Cancer Metastasis Rev. 2015;34(4):735-751.

30. Stehle JR, Jr., Blanks MJ, Riedlinger G, et al. Impact of sex, MHC, and age of recipients on the therapeutic effect of transferred leukocytes from cancer-resistant SR/CR mice. BMC Cancer. 2009;9:328.

31. Sanders AM, Stehle JR, Jr., Blanks MJ, et al. Cancer resistance of SR/CR mice in the genetic knockout backgrounds of leukocyte effector mechanisms: determinations for functional requirements. BMC Cancer. 2010;10:121.

32. Galdiero MR, Bonavita E, Barajon I, et al. Tumor associated macrophages and neutrophils in cancer. Immunobiology. 2013;218(11):1402-1410.

33. Pietenpol JA, Stewart ZA. Cell cycle checkpoint signaling: cell cycle arrest versus apoptosis. Toxicology. 2002;181-182:475-481.

34. Alenzi FQB. Links between apoptosis, proliferation and the cell cycle. Br J Biomed Sci. 2004;61(2):99-102.

35. Colombo MP, Lombardi L, Stoppacciaro A, et al. Granulocyte colony-stimulating factor (G-CSF) gene transduction in murine adenocarcinoma drives neutrophil-mediated tumor inhibition in vivo. Neutrophils discriminate between G-CSF-producing and G-CSF-nonproducing tumor cells. J Immunol. 1992;149(1):113-119.

36. Cavallo F, Giovarelli M, Gulino A, et al. Role of neutrophils and CD4+ T lymphocytes in the primary and memory response to nonimmunogenic murine mammary adenocarcinoma made immunogenic by IL-2 gene. J Immunol. 1992:149(11):3627-3635.

37. Lichtenstein A. Stimulation of the respiratory burst of murine peritoneal inflammatory neutrophils by conjugation with tumor cells. Cancer Res. 1987:47(9):2211-2217.

38. Hicks AM, Willingham MC, Du W, et al. Effector mechanisms of the anti-cancer immune responses of macrophages in SR/CR mice. Cancer Immun. 2006;6:11.

39. Bru A, Souto JC, Alcolea S, et al. Tumour cell lines HT-29 and FaDu produce proinflammatory cytokines and activate neutrophils in vitro: possible 
applications for neutrophil-based antitumour treatment. Mediators Inflamm. 2009;2009:817498.

40. van Spriel AB, van Ojik HH, Bakker A, et al. Mac-1 (CD11b/CD18) is crucial for effective $\mathrm{Fc}$ receptor-mediated immunity to melanoma. Blood. 2003;101(1):253-258

41. van Spriel $A B$, Leusen JH, van Egmond M, et al. Mac-1 (CD11b/CD18) is essential for Fc receptor-mediated neutrophil cytotoxicity and immunologic synapse formation. Blood. 2001;97(8):2478-2486.

42. Metelitsa LS, Gillies SD, Super M, et al. Antidisialoganglioside/granulocyte macrophage-colony-stimulating factor fusion protein facilitates neutrophil antibody-dependent cellular cytotoxicity and depends on FcgammaRII (CD32) and Mac-1 (CD11b/CD18) for enhanced effector cell adhesion and azurophil granule exocytosis. Blood. 2002;99(11):4166-4173.

43. Hubert P, Heitzmann A, Viel S, et al. Antibody-dependent cell cytotoxicity synapses form in mice during tumor-specific antibody immunotherapy. Cancer Res. 2011;71(15):5134-5143.

44. Horner $\mathrm{H}$, Frank $\mathrm{C}$, Dechant $\mathrm{C}$, et al. Intimate cell conjugate formation and exchange of membrane lipids precede apoptosis induction in target cells during antibody-dependent, granulocyte-mediated cytotoxicity. J Immunol. 2007;179(1):337-345.

45. Vadas MA, Nicola NA, Metcalf D. Activation of antibody-dependent cell-mediated cytotoxicity of human neutrophils and eosinophils by separate colony-stimulating factors. J Immunol. 1983;130(2):795-799.

46. Kushner BH, Cheung NK. Absolute requirement of CD11/CD18 adhesion molecules, FcRII and the phosphatidylinositol-linked FcRIII for monoclonal antibody-mediated neutrophil antihuman tumor cytotoxicity. Blood. 1992;79(6):1484-1490

47. Chen ML, Pittet MJ, Gorelik L, et al. Regulatory $\mathrm{T}$ cells suppress tumor-specific CD8 T cell cytotoxicity through TGF-beta signals in vivo. Proc Natl Acad Sci U S A. 2005;102(2):419-424.

48. Mantovani A, Cassatella MA, Costantini $\mathrm{C}$, et al. Neutrophils in the activation and regulation of innate and adaptive immunity. Nat Rev Immunol. 2011;11(8):519-531.

49. Rosales C, Demaurex N, Lowell CA, Uribe-Querol E. Neutrophils: Their Role in Innate and Adaptive Immunity. J Immunol Res. 2016;2016:1469780.

50. Arai H, Gordon D, Nabel EG, et al. Gene transfer of Fas ligand induces tumor regression in vivo. Proc Natl Acad Sci U S A. 1997;94(25):13862-13867.

51. Seino K, Kayagaki N, Okumura K, et al. Antitumor effect of locally produced CD95 ligand. Nat Med. 1997;3(2):165-170.

52. Chen JJ, Sun Y, Nabel GJ. Regulation of the proinflammatory effects of Fas ligand (CD95L). Science. 1998;282(5394):1714-1717.

53. Shimizu M, Fontana A, Takeda Y, et al. Induction of antitumor immunity with Fas/APO-1 ligand (CD95L)-transfected neuroblastoma neuro-2a cells. J Immunol. 1999;162(12):7350-7357.

54. Hohlbaum AM, Moe S, Marshak-Rothstein A. Opposing effects of transmembrane and soluble Fas ligand expression on inflammation and tumor cell survival. J Exp Med. 2000;191(7):1209-1220.

55. Shimizu M, Fontana A, Takeda $Y$, et al. Fas/Apo-1 (CD95)-mediated apoptosis of neutrophils with Fas ligand (CD95L)-expressing tumors is crucial for induction of inflammation by neutrophilic polymorphonuclear leukocytes associated with antitumor immunity. Cell Immunol. 2001;207(1):41-48

56. Behrens CK, Igney FH, Arnold B, et al. CD95 ligand-expressing tumors are rejected in anti-tumor TCR transgenic perforin knockout mice. J Immunol. 2001;166(5):3240-3247.

57. Takamizawa S, Okamoto S, Wen J, et al. Overexpression of Fas-ligand by neuroblastoma: a novel mechanism of tumor-cell killing. J Pediatr Surg. 2000;35(2):375-379.

58. Drozdzik M, Qian C, Lasarte JJ, et al. Antitumor effect of allogenic fibroblasts engineered to express Fas ligand (FasL). Gene Ther. 1998;5(12):1622-1630.

59. Ho MY, Sun GH, Leu SJ, et al. Combination of Fasl and GM-CSF confers synergistic antitumor immunity in an in vivo model of the murine Lewis lung carcinoma. Int J Cancer. 2008;123(1):123-133.

60. Waku T, Fujiwara T, Shao J, et al. Contribution of CD95 ligand-induced neutrophil infiltration to the bystander effect in p53 gene therapy for human cancer. J Immunol. 2000;165(10):5884-5890.

61. Chen YL, Chen SH, Wang JY, et al. Fas ligand on tumor cells mediates inactivation of neutrophils. J Immunol. 2003;171(3):1183-1191.

62. Serrao KL, Fortenberry JD, Owens ML, et al. Neutrophils induce apoptosis of lung epithelial cells via release of soluble Fas ligand. Am J Physiol Lung Cell Mol Physiol. 2001;280(2):L298-305.

63. Hor WS, Huang WL, Lin YS, et al Cross-talk between tumor cells and neutrophils through the Fas (APO-1, CD95)/FasL system: human glioma cells enhance cell viability and stimulate cytokine production in neutrophils. J Leukoc Biol. 2003;73(3):363-368.

64. Desbarats J, Duke RC, Newell MK. Newly discovered role for Fas ligand in the cell-cycle arrest of CD4+ T cells. Nat Med. 1998;4(12):1377-1382.

65. Rescigno M, Piguet V, Valzasina B, et al. Fas engagement induces the maturation of dendritic cells (DCs), the release of interleukin (IL)-1beta, and the production of interferon gamma in the absence of IL-12 during DC-T cell cognate interaction: a new role for Fas ligand in inflammatory responses. J Exp Med. 2000;192(11):1661-1668. 